\title{
First printed 3D heart model based on cardiac magnetic resonance imaging data in Slovakia
}

\author{
Olejnik $\mathrm{P}^{1,2}$, Juskanic $\mathrm{D}^{3}$, Patrovic $\mathrm{L}^{3}$, Halaj $\mathrm{M}^{3}$ \\ Department of Pediatric Cardiology, Pediatric Cardiac Centre, National Institute of Cardiovascular Diseases, \\ Bratislava, Slovakia. petoolejnik@gmail.com
}

\section{ABSTRACT}

OBJECTIVES: To present our experience with initial fabrication of 3D printed model of heart from cardiac magnetic resonance $(C M R)$ imaging data.

METHODS: A 20-year-old patient with a congenital heart defect after a surgical correction underwent CMR imaging. A novel whole-heart CMR imaging sequence, not requiring gadolinium contrast material application, was performed. Image data from this CMR sequence were used for 3D heart model printing.

RESULTS: A life-like 3D printed copy of the heart with a congenital defect with superior visualization of cardiovascular anatomical details was successfully fabricated.

CONCLUSION: 3D printing of the heart copies from novel whole heart CMR imaging data is feasible and harmless to patients. These models can be used for operative planning of complex congenital heart defects (Fig. 4, Ref. 14). Text in PDF www.elis.sk.

KEY WORDS: 3D printing, heart, model, cardiac magnetic resonance.

\section{Introduction}

3D medical model printing has been primarily used in maxillofacial and orthopedic surgery $(1,2)$. The models were described as extremely helpful for operative planning. Later, 3D printed heart models based on computed tomography (CT) or cardiac magnetic resonance (CMR) imaging started to be used for operative planning in pediatric cardiology owing to the superior quality in complex congenital heart defects imaging $(3,4,5,6)$. Until now, as presented in our previous study, only CT images have been used as the input data for 3D cardiac model printing in Slovakia (7). The drawback of this type of data acquisition lies in inevitable radiation exposure of the patient, as well as potential harmful effect of iodine contrast material on kidneys and thyroid gland. The standard CMR anatomical imaging including intravenous gadolinium contrast material application represents some potential risk for the patient too because gadolinium exposure can lead to the development of nephrogenic systemic fibrosis (8).

Therefore, the goal of our study was to fabricate a 3D printed model of a heart with a congenital heart defect, in which the input data for the 3D printer would be acquired from novel wholeheart CMR imaging sequence not requiring gadolinium contrast

${ }^{1}$ Department of Pediatric Cardiology, Pediatric Cardiac Centre, National Institute of Cardiovascular Diseases, Bratislava, Slovakia, ${ }^{2}$ Department of Pediatric Cardiology, Faculty of Medicine, Comenius University, Bratislava, Slovakia, and ${ }^{3}$ Jessenius Diagnostic Centre, Nitra, Slovakia

Address for correspondence: P. Olejnik, MD, PhD, Department of Pediatric Cardiology, Pediatric Cardiac Centre, National Institute of Cardiovascular Diseases, Limbova 1, SK-833 51 Bratislava, Slovakia

Phone: +421.907152641 material application. It is worthwhile noting that the latter type of procedure has not been performed in Slovakia so far.

\section{Methods and results}

A female patient with tetralogy of Fallot (TOF) after complete surgical correction including pulmonary valve trans-annular patch underwent imaging of the heart by CMR (SIEMENS Magnetom Skyra 3,0T, Erlangen, Germany) at the age of 20 years. Patients with this condition suffer from severe pulmonary regurgitation leading to RV dilatation and dysfunction. Therefore, the goal of CMR imaging in such patients is to evaluate volumetric and functional parameters of the right ventricle by using standard CMR imaging protocol

To achieve superior quality of volumetric input data for the 3D printer, a novel ultra-fast 3D whole-heart sequence, not requiring intravenous gadolinium contrast material application, was used in our patient too. It is a T2 prepared gradient echo sequence exploiting fast low-angle shot technique with cartesian readout of k-space. The parameters of repetition time, echo time, slice thickness, oversampling, field of view, distance factor, and preparation duration were $329.37 \mathrm{~ms}, 1.48 \mathrm{~ms}, 1 \mathrm{~mm}, 20 \%, 320 \mathrm{~mm}, 20 \%$, and 50 $\mathrm{ms}$, respectively, while flip angle increased to $20^{\circ}$ improved tissue contrast and cavity-endocardium interface delineation. Respiratory navigation, as well as electrocardiography triggering, had to be used during data acquisition in order to reduce heart pulsation and breathing artefacts. The consequent trade-off in terms of prolonged examination time and decreased signal to noise ratio was compensated for by advantage of using a 3T magnetic field strength. As a result, mean scanning time up to 8 minutes was achieved, which 

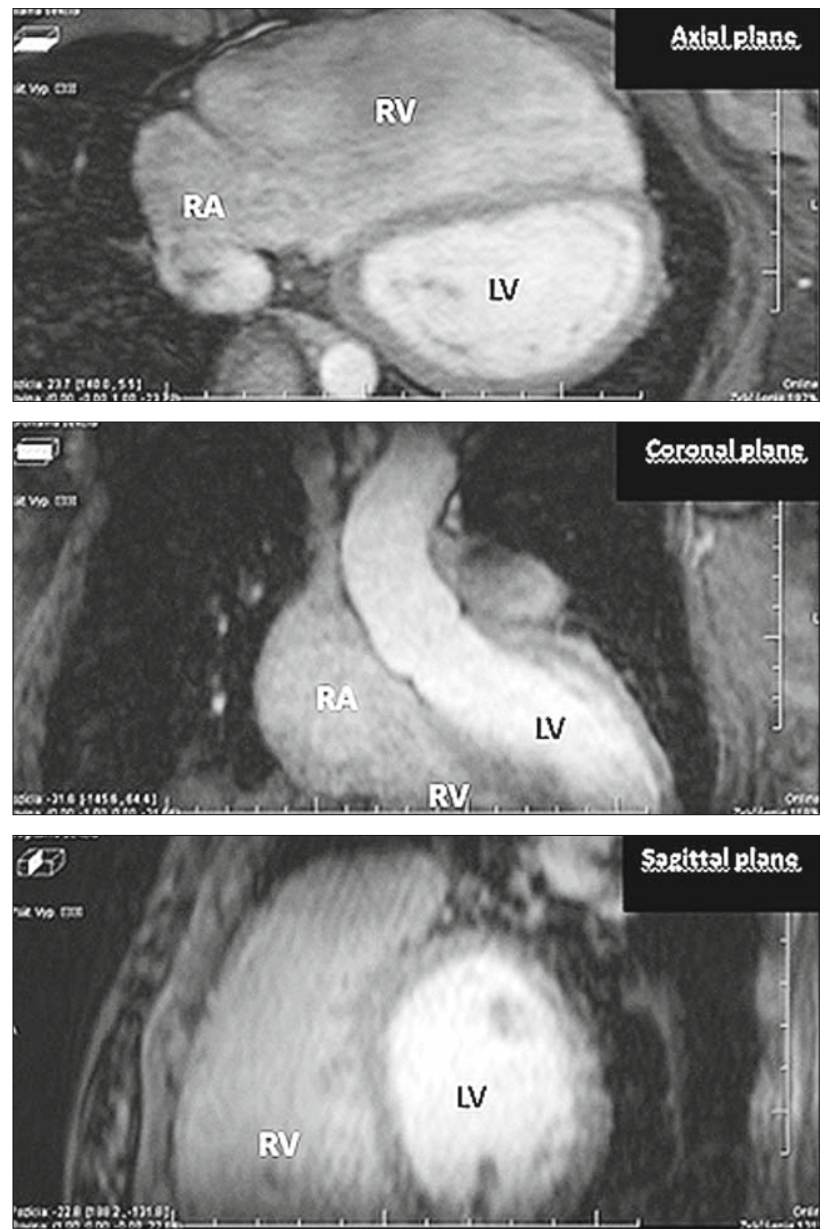

Fig. 1. CMR images of the heart acquired from novel whole-heart sequence in different planes. Bright (hyperintensive) areas represent lumens of cardiac chambers and vessels. Dark (hypointensive) areas represent walls of cardiac chambers and vessels. $R V$ - right ventricle, RA - right atrium, $L V$ - left ventricle.

is clinically feasible. A final high-quality stack of axial slices of the thorax with $1.1 \mathrm{~mm}$ slice thickness was produced. The image data acquired from this unique CMR sequence were stored in DICOM format (Fig. 1). An open source software package "3D slicer 4.3" was used for DICOM data processing. Firstly, the grey-scale manipulation increased the contrast boundaries of endocardium separating the blood pool from myocardium/vessel walls. The image segmentation continued with the extraction of labeled region of interest (ROI), i.e. blood pool from myocardium/vessel walls (Fig. 2). After ROI extraction, the model surface was smoothed, and model data were triangulated and stored in stereolithography file format (Fig. 3). These data were recognized by the 3D printing device “Zortrax M200” (Zortrax S.A., Olsztyn, Poland). The additive technique of fusion deposition modelling was employed for printing a 1:1 life-like 3D copy of the heart. Printing filament “Z-ABS”compatible with "Zortrax M200“ printer was selected for model fabrication. The width of one layer in vertical scale was $0.19 \mathrm{~mm}$ which provided an optimal ratio between both velocity and spatial accuracy of printing. Total printing time for this model
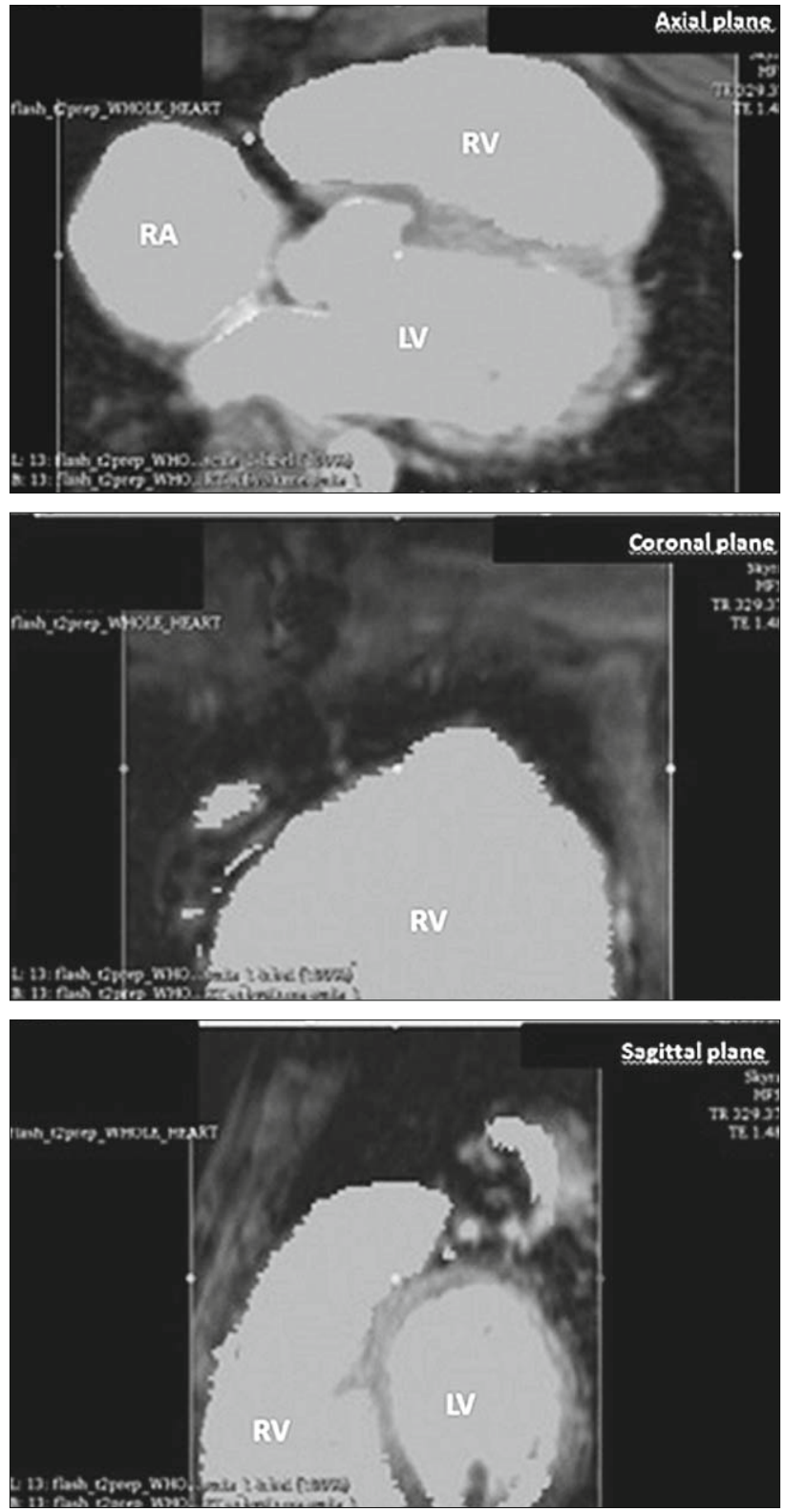

Fig. 2. 3D image data set segmentation in "3D slicer 4.3". Region of interest (lumens of cardiac chambers and vessels) labeling with grey color in basic planes. $\mathrm{RV}$ - right ventricle, $\mathrm{RA}$ - right atrium, LV left ventricle.

was 46 hours and 25 minutes. An amount of 263 grams of filament was utilized for the whole exemplar fabrication. Once the 3D model printing was completed, the supporting material had to be removed manually. When holding the printed heart model in the hand, one can appreciate the dilated right ventricle outflow tract, as well as proximal left pulmonary artery branch stenosis (Fig. 4).

\section{Discussion}

Analyses of printed 3D heart replicas bring the real 3D tactile experience with the possibility of detailed examination from 


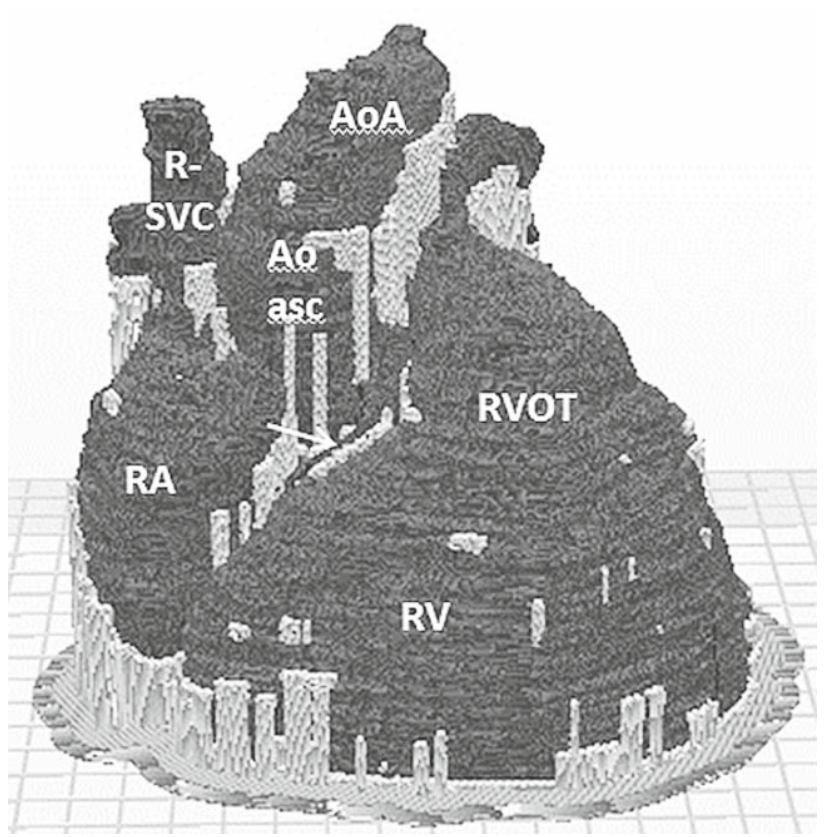

Fig. 3. Heart model in stereolithography file format. Frontal view. Dark areas represent segmented lumens of cardiac chambers and vessels. Bright areas represent calculated support material, which makes the model solid during the printing process. RV - right ventricle, RA right atrium, $L V$ - left ventricle, $R V O T$ - right ventricle outflow tract, Ao asc - ascending aorta, AoA - aortic arch, R-SVC - right superior caval vein, arrow- right coronary artery.

all possible perspectives $(5,9,10)$. This kind of information is helpful for surgical decision-making, as even minor anatomical details can indicate the optimal surgical approach in each individual patient with a complex congenital heart defect. Hence, the use of 3D heart models allows one to avoid unexpected findings in vivo, and thus potentially reduce the procedural time and morbidity/mortality. $(7,11)$.

The analysis of 3D heart models in TOF patients after complete surgical correction, either by a cardiac surgeon or interventional cardiologist, improves the understanding of various types of right ventricular outflow tracts, which then allows better planning of either surgical or transcatheter pulmonary valve replacement (12). Owing to the convenience of 3D heart printing it was possible to choose the optimal approach in our patient too.

Data for 3D medical model printing, including pediatric cardiology, are mostly based on CT or CMR imaging $(5,13,14)$. As we did not use CT-angiography, and at the same time, we acquired CMR image data from novel whole-heart sequence not requiring gadolinium application, our patient was prevented from all potential risks such as contrast nephropathy, thyroid gland damage, as well as from nephrogenic systemic fibrosis. Based upon this successful initial fabrication of a 3D printed heart model, we ascertained that the process of printing heart copies from CMR imaging data is feasible, harmless to patients, and can be used for operative planning of complex congenital heart defects in the future.

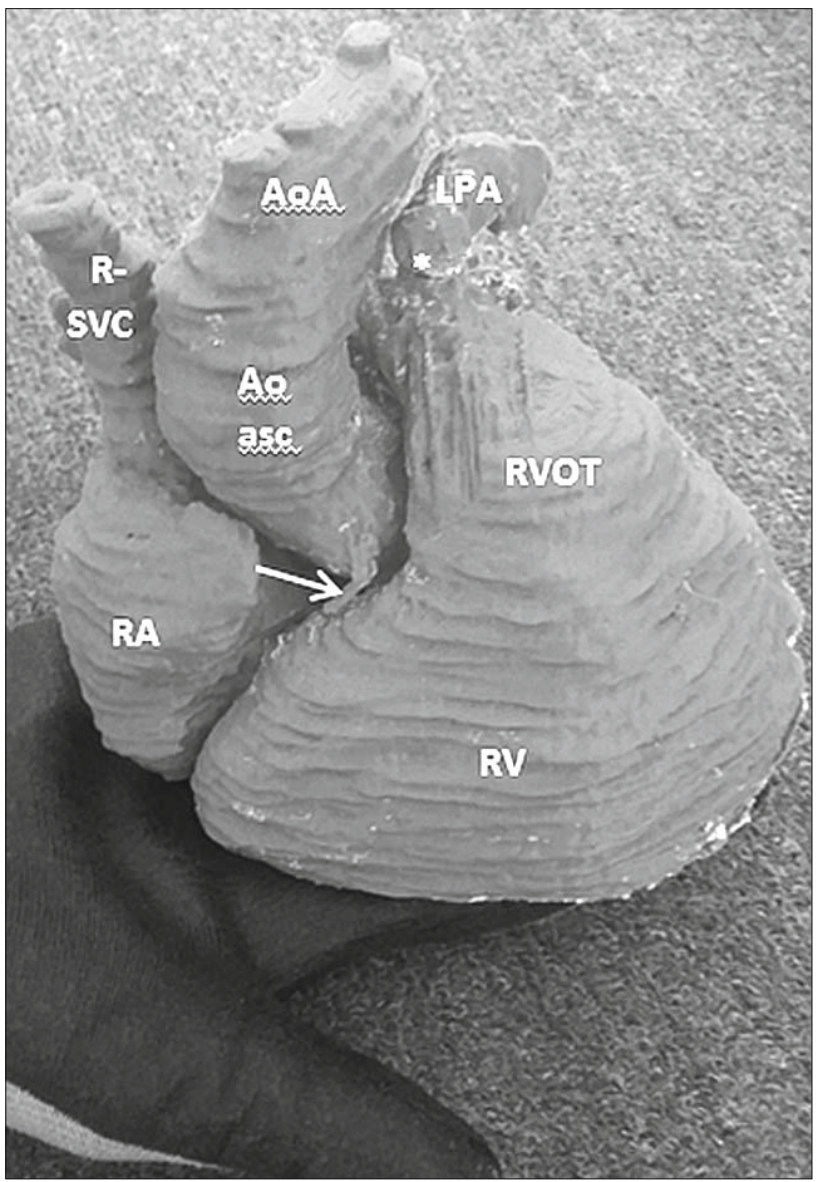

Fig. 4. 3D printed heart model held in hand of a physician. Right upper view. $R V$ - right ventricle, RA - right atrium, RVOT - right ventricle outflow tract (dilated), Ao asc - ascending aorta, AoA - aortic arch, R-SVC - right superior caval vein, arrow - right coronary artery, asterisk - proximal left pulmonary artery branch stenosis.

\section{Learning points}

Input data for fabricating 3D printed models of complex congenital heart defects can be acquired by use of novel whole-heart CMR sequence which does not require gadolinium administration. A high-quality spatial resolution of achieved image data enables to print real life-like 1:1 copies of the hearts with superior visualization of cardiovascular anatomical details. These models can be used for operative planning in pediatric cardiology.

\section{References}

1. D 'Urso PS, Barker TM, Earwaker WJ, Bruce LJ, Atkinson RL, Lanigan MW. Stereolithographic biomodelling in cranio-maxillofacial surgery: a prospective trial. J Craniomaxillofac Surg 1999; 27: 30-37.

2. Fleming ME, Waterman SS, Lewandowski LR, Chi BB. Use of 3-dimensional stereolithographic polymer models for heterotopic ossification surgical excision. Orthopedics 2013; 36: 282-286. doi: 10.3928/0147744720130327-06. 


\section{1-784}

3. Valverde I, Gomez G, Suarez-Mejias C et al. 3D printed cardiovascular models for surgical planning in complex congenital heart diseases. J Cardiov Magn Reson 2015; 17 (Suppl 1): 196. doi: 10.1186/1532-429X17-S1-P196.

4. M Lau I, Sun Z. Three-dimensional printing in congenital heart disease: A systematic review. J Med Radiat Sci 2018; 3: 226-236. doi: org/10.1002/ jmrs.268.

5. Schmauss D, Haeberle S, Hagl Ch, Sodian R. Three-dimensional printing in cardiac surgery and interventional cardiology: a single-centre experience. Eur J Cardiothorac Surg 2015; 47: 1044-1052. doi: 10.1093/ ejcts/ezu310.

6. Balegadde AV, Vijan V, Thachathodiyl R, Kappanayil M. A case of asymptomatic large aortopulmonary window in an adult: Role of cardiac CT, CMRI, and 3D printing technology. Anatol J Cardiol 2018; 19 (1): 72-74. doi: 10.14744/AnatolJCardiol.2017.7948.

7. Olejník P, Nosal M, Havran T et al. Utilisation of three-dimensional printed heart models for operative planning of complex congenital heart defects. Kardiol Pol 2017; 75 (5): 495-501. doi: 10.5603/KP.a2017.0033.

8. Deo A, Fogel M, Cowper SE. Nephrogenic systemic fibrosis: a population study examining the relationship of disease development to gadolinium exposure. Clin J Am Soc Nephrol 2007; 2: 264-267.
9. Moore RA, Riggs KW, Kourtidou S et al. Three-dimensional printing and virtual surgery for congenital heart procedural planning. Birth Defects Res 2018; 110 (13): 1082-1090. doi: 10.1002/bdr2.1370.

10. Lau IWW, Liu D, Xu L, Fan Z, Sun Z. Clinical value of patientspecific three-dimensional printing of congenital heart disease: Quantitative and qualitative assessments. PLoS One 2018; 13: e0194333. doi: org/10.1371/journal.pone.0194333.

11. Valverde I, Gomez G, Gonzalez A et al. Three-dimensional patientspecific cardiac model for surgical planning in Nikaidoh procedure. Cardiol Young 2015; 25: 698-704. doi: 10.1017/S1047951114000742.

12. Ntsinjana HN, Hughes ML, Taylor AM. The Role of Cardiovascular Magnetic Resonance in Pediatric Congenital Heart Disease. J Cardiovasc Magn Reson 2011; 13: 51.

13. Foley TA, El Sabbagh A, Anavekar AN et al. 3D-Printing: Applications in Cardiovascular Imaging. Curr Radiol Rep 2017; 5: 43. Doi: org/10.1007/s40134-017-0239-3.

14. Kiraly L. Three-dimensional modelling and three-dimensional printing in pediatric and congenital cardiac surgery. Transl Pediatr 2018; 7 (2): 129-138. doi: 10.21037/tp.2018.01.02.

Received September 5, 2018. Accepted October 8, 2018. 